\title{
MHD TURBULENCE IN THE SOLAR WIND AND INTERPLANETARY DYNAMO EFFECTS
}

\author{
E. MARSCH AND C.-Y.TU* \\ Max-Planck-Institut für Aeronomie, \\ W-3411 Katlenburg-Lindau, Germany \\ * also at Department of Geophysics, Peking University, Beijing, China
}

ABSTRACT. From the fluctuations of the velocity and magnetic field observed in different kinds of solar wind the fluctuating electric fields are derived, and their power spectra are constructed and analysed. The mean electromotive force $\mathcal{E}$ generated by the turbulent motions depends upon the nature of the fluctuations. Simple dynamo theory predicts a linear relationship between $\mathcal{E}$ and the mean magnetic field $\mathbf{B}_{0}$. Correlation studies carried out to establish the alpha effect in the solar wind have given negative results.

\section{Introduction}

Fluctuations of magnetic field $(\delta \mathbf{B})$ and velocity $(\delta \mathbf{V})$ in the solar wind have been investigated in much detail and quite successfully described in terms of MHD turbulence phenomenology and theory (for recent reviews see e.g. Marsch, 1991; Mangeney et al., 1991; Roberts and Goldstein, 1991). Whereas much effort has been spent on analysing and understanding the characteristics and origins of the fluctuations and their energy and helicity spectra, almost no attention has been payed to the turbulent electric fields and their power spectra, let alone to the question of a possible turbulent dynamo in the interplanetary medium. The present paper will briefly address this problem. The effect that the pressure of Alfvénic fluctuations has on the background solar wind flow has been thoroughly studied (e.g. in the reviews by Leer et al., 1982; Marsch, 1993), and its major role in the acceleration of high-speed flows is generally accepted. Likewise, one expects an electromotive force

$$
\mathcal{E}=<\delta \mathbf{V} \times \delta \mathbf{B}>
$$

to be associated with the fluctuations. This electric field shows up in the induction equation in addition to the mean convection electric 
field, a result that has been worked out within the framework of meanfield electrodynamics and dynamo theory, as they are described in the classical monographs by Moffett (1978) and Krause and Rädler (1980).

\section{MHD fluctuations and the electromotive force}

Mean-field electrodynamics is based on the assumpion that a two-scale decomposition of the flow velocity and magnetic field is meaningful, in such a way that the average magnetofluid can be described by mean fields varying on large temporal and spatial scales. Superposed on these fields are small-scale random field components that strongly vary on small scales, being of the order of the correlation time and length of the turbulence. Thus we decompose the fields of the magnetofluid such that $\mathbf{V}=\mathbf{V}_{0}+\delta \mathbf{V}$ and $\mathbf{B}=\mathbf{B}_{0}+\delta \mathbf{B}$, whereby $\mathbf{B}_{0}=\langle\mathbf{B}\rangle$ and $\mathbf{V}_{0}=\langle\mathbf{V}\rangle$. The mean fields are given by appropriate ensemble averages (for a discussion of this subject in the solar wind context see the papers by Matthaeus et al., 1986, and Matthaeus and Goldstein, $1982 \mathrm{a}, \mathrm{b})$, which are here calculated as time averages.

By ensemble averaging the momentum and induction equations only terms of second order in the fluctuations will survive. These are the turbulent stresses and the electromotive force $\mathcal{E}$, as given by expression (1). For the sake of simplicity we assumed an incompressible plasma in this derivation. Notice that for pure Alfvénic fluctuations the correlation holds: $\delta \mathbf{V}= \pm \delta \mathbf{B} / \sqrt{4 \pi \rho_{0}}$. Consequently, the stress tensor reduces to the scalar Alfvén wave pressure, and there is no turbulent electric field, since the fluctuations of velocity and magnetic field are aligned. In general, however, the full stress tensor has to be added to the kinetic and magnetic stresses of the mean flow. Here we shall only consider the mean-field induction equation, reading

$$
\frac{\partial}{\partial t} \mathbf{B}_{0}=\nabla \times\left(\mathbf{V}_{0} \times \mathbf{B}_{0}\right)+\nabla \times \mathcal{E}+\eta \nabla^{2} \mathbf{B}_{0}
$$

where the magnetic diffusivity is denoted by $\eta$. Note that due to rare collisions in the solar wind the classical diffusivity is numerically rather small and its applicability is questionable (Montgomery, 1983). For anomalous transport processes see the recent review by Marsch (1991). 
The turbulent EMF also affects the spatial and temporal evolution of the spectral energy of the fluctuations. Theoretical models and spectral transfer equations have recently been proposed by various authors (Zhou and Matthaeus, 1990a,b; Marsch and Tu, 1989; Tu and Marsch, 1990) to describe the fluctuations in terms of the Elsässer fields $\mathbf{Z}^{ \pm}=\mathbf{V} \pm \mathbf{V}_{A}$, where $\mathbf{V}_{A}=\mathbf{B} / \sqrt{4 \pi \rho}$ is the Alfvén velocity. The electromotive force $\mathcal{E}$ can be expressed (see Marsch and $\mathrm{Tu}$ (1992) for more details) by the non-diagonal elements of the correlation tensor, which in spectral transfer equations are associated with turbulence excitation by spatial gradients and the shear of $\mathbf{V}_{0}$ and $\mathbf{B}_{0}$ or $\mathbf{Z}_{0}^{+}$and $\mathbf{Z}_{0}^{-}$. These source terms play, besides nonlinearities, a leading role in the evolution of the specific turbulent energies

$$
e^{ \pm}=\frac{1}{2}<\left(\delta \mathbf{Z}^{ \pm}\right)^{2}>
$$

of the Elsässer fields. Comprehensive interplanetary studies (see the above cited reviews) on the phenomenology and spectral and spatial characteristics of $e^{ \pm}$exist, and also numerical simulations (e.g. Grappin et al., 1982,1983; Roberts et al., 1992) have been carried out to understand their nonlinear evolution. Lack of space prohibits us to discuss these any further. We shall therefore concentrate on a few selected observational results.

\section{Turbulent electric field spectra}

Tu et al. (1989) and Grappin et al. (1990) have shown that the $e^{ \pm}$ spectra do systematically vary as a function of the solar wind stream structure during the period of solar activity minimum. Similarly, the spectra of electric field fluctuations vary when calculated by means of the Fourier transforms of the autocorrelation function according to equation (1). Generally speaking, the spectra of $e^{ \pm}$are flatter in fast streams and more fully developed toward a Kolmogorov-type spectrum in the slow wind associated with the heliospheric current sheet. Of course the $\mathcal{E}$ spectra by definition are not positive definite but oscillate in sign as a function of frequency or wavevector. However, the modular envelopes show spectral features and evolutionary trends similar to the $e^{ \pm}$spectra. 


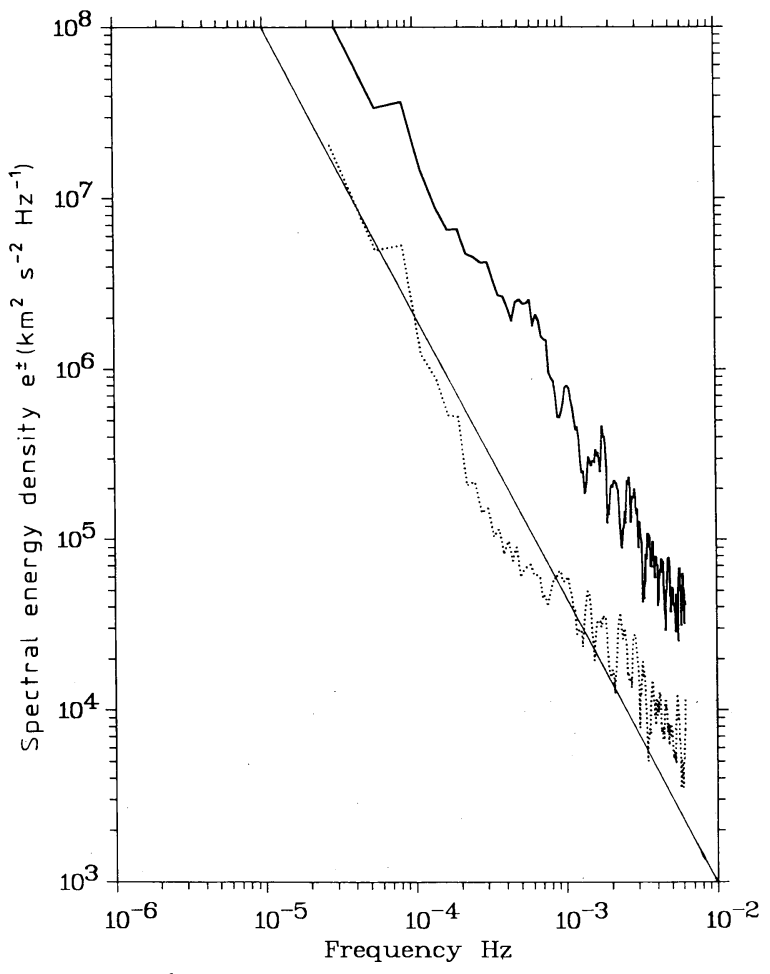

Figure 1: Spectral energy density of the magnetohydrodynamic fluctuations in terms of Elsässer variables plotted versus frequency. The dominance of $e^{+}$over $e^{-}$indicates that Alfvénic fluctuations prevail for this time period of Helios 1 data from 1980 obtained at $0.53 \mathrm{AU}$.

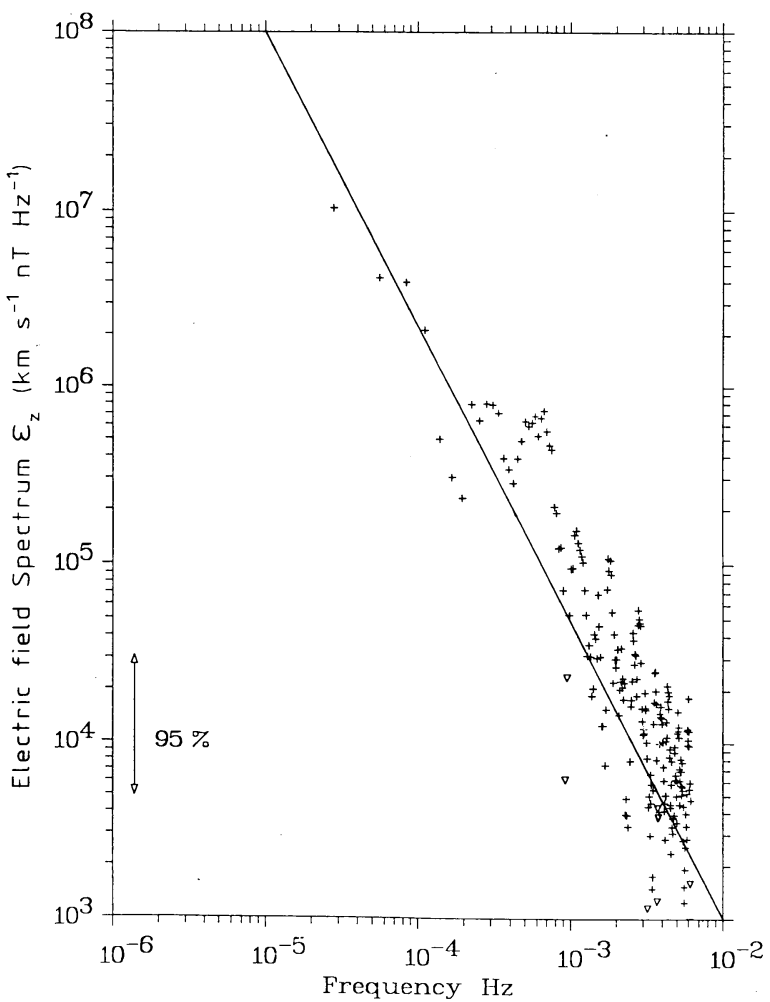

Figure 2: The out-ofecliptic component $\mathcal{E}_{z}$ of the ponderomotive electric field versus frequency. The reference line corresponds to a Kolmogorov spectrum with a slope of $5 / 3$. 
In Fig. 1 we show representative energy spectra for a fast solar wind $(637 \mathrm{~km} / \mathrm{s})$ near solar maximum in 1980 from Helios observations at 0.53 AU. Clearly $e^{+}$dominates $e^{-}$, indicating Alfvénic fluctuations with a developed turbulent cascade and spectral index close to the $-5 / 3$ value. For pure Alfvén waves $\mathcal{E}$ after definition (1) should vanish. This is not the case, however, in the in-situ observations, where always a small amount of $\delta \mathbf{Z}^{-}$exists, which yet is sufficient to establish first a nonlinear cascade and second a nonzero turbulent electric field. The corresponding spectrum of the z-component of $\mathcal{E}$ is given in Fig. 2, showing $\mathcal{E}_{z}$ versus frequency in the range from $10^{-5}$ to $10^{-2}$ $\mathrm{Hz}$. Note that crosses indicate positive values and up-side-down triangles negative values. Apparently, there is substantial scatter in the data, but the overall shape of the spectrum is near a Kolmogorov one. Whereas the $\mathcal{E}_{z}$ field components are predominantly positive, this is quite different for the $\mathcal{E}_{y}$ spectrum (not shown here), which also has a $-5 / 3$ spectral envelope but strongly alternates in sign, particularly at frequencies higher than some $10^{-4} \mathrm{~Hz}$. In slow solar wind one usually finds $e^{+} \gtrsim e^{-}$(see e.g. Marsch and Tu, 1990) and the fluctuation level is considerably lower than in fast streams. Also the turbulent electric field is generally smaller in slow wind.

\section{Dynamo theory and test of the alpha effect}

As emphasized in the introduction the electromotive force $\mathcal{E}$ is of central importance in dynamo theory (Krause and Rädler, 1980). One of the key questions is whether $\mathcal{E}$ can be expressed in terms of the mean fields in such a way that closure of the induction equation is obtained in combination with the fluid equation of motion. The linearity of the induction equation in the fluctuations and mean magnetic field implies that $\mathcal{E}$ be a linear functional of $\mathbf{B}_{0}$. Generally, the functional expansion coefficients are pseudo-tensors of different ranks. If the turbulence is statistically isotropic and homogeneous then one has the only possible form:

$$
\mathcal{E}=\alpha \mathbf{B}_{0}-\beta \nabla \times \mathbf{B}_{0}
$$

The alpha effect is based on the parameter $\alpha \sim<\delta \mathbf{V} \cdot(\nabla \times \delta \mathbf{V})>$ and thus depends on kinetic helicity. It therefore requires that reflexional symmetry is broken, since otherwise $\alpha$ would vanish identically. For 
isotropic turbulence the induction equation can then be written (Moffatt and Tsinober, 1992) with a scalar alpha coefficient and turbulent diffusivity $\beta$ as follows:

$$
\frac{\partial \mathbf{B}_{0}}{\partial t}=\alpha \nabla \times \mathbf{B}_{0}+(\eta+\beta) \nabla^{2} \mathbf{B}_{0}
$$

We have analysed the data with respect to possible correlations between $\mathcal{E}$ and $\mathbf{B}_{0}$ and thereby investigated quite a few different periods of solar wind flows, but did not find any apparent linear correlation. These negative findings put the existence of a scalar alpha effect in question, a result that is corroborated in Figs. 3 and 4 of Marsch and $\mathrm{Tu}$ (1992), showing detailed correlation plots between the 1-hour averages of the cartesian components of $\mathbf{B}_{0}$ and $\mathcal{E}$. In conclusion, there seems to be no indication of a linear relationship. To make shure this was also true for other sampling periods we repeated this study at a 10-hour scale with the same negative conclusion. In this context it is important to note that Marsch and Tu (1990) already established in the spectral analysis of the Elsässer fields that solar wind turbulence was locally mirror-symmetric, in which case $\alpha$ had of course to be strictly zero.

\section{Conclusions}

The purpose of this short contribution was to investigate electric field fluctuations in the solar wind and to scrutinize the data with respect to possible turbulent dynamo effects in the interplanetary medium. Electric field fluctuations have been studied in the framework of mean-field electrodynamics. A sizable EMF has indeed been found in various types of solar wind flow, with $\mathcal{E}$ being particularly strong in Alfvénic fluctuation periods with $e^{+}>e^{-}$. The electric field spectra show signatures of fully developed turbulence, in particular when the fluctuations are intense and occur in the slow wind. In correlation studies searching for a scalar alpha effect we could not establish a simple linear relationship between $\mathcal{E}$ and $\mathbf{B}_{0}$.

\section{References}

Grappin, R., U. Frisch, J. Léorat, and A. Pouquet, Astron. Astrophys., $105,6(1982)$ 
Grappin, R., A. Pouquet, and J. Léorat, Astron. Astrophys., 126, 51 (1983)

Grappin, R., A. Mangeney, and E. Marsch, J. Geophys. Res., 95, 8197 (1990)

Krause, F., and K.-H. Rädler, Mean-field magnetohydrodynamics and dynamo theory, Akademie-Verlag, Berlin (1980)

Leer, E., T.E. Holzer, and F. Flå, Space Sci. Rev., 33, 161 (1982)

Mangeney, A., R. Grappin, and M. Velli, in Advances in Solar System Magnetohydrodynamics, ed. by E.R. Priest (1990)

Marsch, E., in Physics of the Inner Heliosphere, vol. 2, ed. by R. Schwenn and E. Marsch, Springer-Verlag, Berlin, Heidelberg, New York, 45 and 159 (1990)

Marsch, E., Adv. Space Res., in press, (1993)

Marsch, E., and C.-Y. Tu, J. Plasma Phys., 41, 479 (1989)

Marsch, E., and C.-Y. Tu, J. Geophys. Res., 90, 95 (1990)

Marsch, E., and C.-Y. Tu, in Solar Wind Seven, ed. by E. Marsch and R. Schwenn, Pergamon Press, Oxford, 505 (1992)

Matthaeus, W.H., and M.L. Goldstein, J. Geophys. Res., 87, 6011 (1982)

Matthaeus, W.H., and M.L. Goldstein, J. Geophys. Res., 87, 10347 (1982)

Matthaeus, W.H., M.L. Goldstein, and J.H. King, J. Geophys. Res., 91, 59 (1986)

Moffatt, H.K., Magnetic Field Generation in Electrically Conducting Flu$i d s$, Cambridge University Press, Cambridge (1978)

Moffatt, H.K., and A. Tsinober, Annu. Rev. Fluid Mech. 24, 281 (1992)

Montgomery, D.C., in Solar Wind Five, ed. by M. Neugebauer, NASA CP-2280, 107 (1983)

Roberts, D.A., and M.L. Goldstein Reviews of Geophysics, Supplement, 932 (1991)

Roberts, D.A., M.L. Goldstein, W.H. Matthaeus, and S. Gosh, J. Geophys. Res. 97, 17115 (1992)

Tu, C.-Y., E. Marsch, and K.M. Thieme, J. Geophys. Res., 94, 739 (1989)

Tu, C.-Y., and E. Marsch, J. Plasma Phys., 44, 103 (1990)

Velli, M., R. Grappin, and A. Mangeney, in Plasma Phenomena in the Solar Atmosphere, ed. by M. A. Dubois, Editions de Physique, Orsay (1989)

Zhou, Y., and W.H. Matthaeus, J. Geophys. Res., 95, 14863 (1990)

Zhou, Y., and W.H. Matthaeus, J. Geophys. Res., 95, 14881 (1990) 